\title{
APPLYING SPECIES-SENSITIVITY DISTRIBUTIONS IN ECOLOGICAL RISK ASSESSMENT: ASSUMPTIONS OF DISTRIBUTION TYPE AND SUFFICIENT NUMBERS OF SPECIES
}

\author{
Michael C. Newman,* David R. Ownby, Laurent C.A. Mézin, David C. Powell, \\ Tyler R.L. Christensen, Scott B. Lerberg, and Britt-Anne Anderson \\ College of William and Mary, Virginia Institute of Marine Science, Gloucester Point, Virginia 23062-1346, USA
}

(Received 13 Janaury 1999; Accepted 6 June 1999)

\begin{abstract}
Species-sensitivity distribution methods assemble single-species toxicity data to predict hazardous concentrations (HCps) affecting a certain percentage (p) of species in a community. The fit of the lognormal model and required number of individual species values were evaluated with 30 published data sets. The increasingly common assumption that a lognormal model best fits these data was not supported. Fifteen data sets failed a formal test of conformity to a lognormal distribution; other distributions often provided better fit to the data than the lognormal distribution. An alternate bootstrap method provided accurate estimates of HCp without the assumption of a specific distribution. Approximate sample sizes producing HC5 estimates with minimal variance ranged from 15 to 55, and had a median of 30 species-sensitivity values. These sample sizes are higher than those suggested in recent regulatory documents. A bootstrap method is recommended that predicts with $95 \%$ confidence the concentration affecting $5 \%$ or fewer species.
\end{abstract}

Keywords—Risk assessment Species sensitivity Bootstrap Statistics Sample size

\section{INTRODUCTION}

Concentration-effect data applied to ecological risk assessment usually come from single-species toxicity tests measuring effects to individuals. However, populations, communities, and ecosystems are generally the entities to be protected. To resolve this incongruity between individual-based data and the complex biological entities addressed in ecological risk assessment, an evaluation of species-sensitivity distributions has been proposed [1]. Single-species test data are combined to predict concentrations affecting only a certain percentage of species in a community. Single-species data (e.g., median lethal concentration [LC50] or no-observed-effect concentration [NOEC] values) for many species are fit to a distribution such as the lognormal or log-logistic. From this distribution of species sensitivities, a hazardous concentration ( $\mathrm{HCp})$ is identified at which a certain percentage (p) of all species is assumed to be affected. The most conservative form of this approach uses the lower $95 \%$ tolerance limit of the estimated percentage to ensure that the specified level of protection is achieved [1-3].

Species-sensitivity distribution or extrapolation methods are being incorporated into assessments of ecological risk [4,5] and into recommendations for pesticide registration [6]. A general species-sensitivity distribution approach is detailed in the new U.S. Environmental Protection Agency guidelines for ecological risk assessment [7]. The variants most recently espoused specify a lognormal distribution for species sensitivities regardless of whether the modeled effect metrics were median effective concentration (EC50), LC50, NOEC, or maximum acceptable toxicant concentration values [1-5].

The species-sensitivity distribution innovation carries as-

* To whom correspondence may be addressed (newman@vims.edu). Contribution 2223 of the College of William and Mary's Virginia Institute of Marine Science and School of Marine Science. sumptions needing scrutiny [1]. The following are seven of the most important concerns.

1. The LC50, EC50, NOEC, and maximum acceptable toxicant concentration have very significant deficiencies as measures of effect to field populations and communities [8]. Any secondary metric based on such compromised metrics possesses the same deficiencies.

2. Whether any species loss is acceptable is equivocal. Proponents of the species-sensitivity distribution approach argue that enough redundancy exists in communities to allow some loss [9]. The counterpoint to this redundant species hypothesis combines the rivet popper hypothesis (community integrity is reduced by each loss of a species) with the argument that a conservative stance is best when faced with uncertainty in ecological risk assessment [10]. The rivet popper hypothesis is supported by the few studies directly addressing the question of redundancy [10-14]. Ecosystem productivity, stability, sustainability, and nutrient retention decrease as species diversity decreases, although species loss up to a certain level seemed to have little influence on drought resistance or resilience of prairie grassland communities [12-14].

3 . If performed without thought, species-sensitivity distribution methods could discount the importance of maintaining dominant and keystone species [15], and the influence of species interactions [8].

4. In situ exposure differs among species because of dissimilarities in behavior, feeding habits, life histories, life stages, and microhabitats. Exposure differences proscribe the direct application of concentration-effect data derived by singlespecies laboratory testing [16] in this or other risk assessment procedures.

5. A bias exists toward mortality data despite the plausibility of nonlethal effects being at least as important in determining local population extinction. Also, a bias exists to- 
ward a subset of standard species that are amenable to laboratory culture and manipulation. However, these shortcomings are shared by other risk assessment applications of ecotoxicity data.

6. The assumption of a specific distribution may not be justifiable for many data sets, which, if plotted alongside values predicted from the distribution, show clear deviations from the assumed lognormal distribution. Because data for diverse taxonomic groups are pooled, even the basic assumption of a unimodal distribution is dubious. Although the present movement toward using specific taxonomic groups in this speciessensitivity distribution procedure may reduce the magnitude of this problem, the ambiguity associated with selecting the lognormal distribution will remain.

7. Most discussions of appropriate sample size and sample representativeness of the community are focused on practical issues of producing an agreeable number of observations for regulatory agencies [6]. The exception was the original work of Kooijman [1], who introduced the approach of applying parametric methods to formally address the sample size question. Assessment of these issues of sufficient sample size and representativeness is difficult in most applications. Most studies either do not report confidence limits along with estimates of $\mathrm{HCp}$, fail to calculate minimal sample sizes, or do not discuss the completeness of the data set relative to the community at risk.

Bootstrap estimation provides a partial answer to two of the above issues, ambiguity in selecting a specific distribution and estimation of the approximate number of species needed to precisely estimate HCp [17]. Bootstrap estimation alleviates the difficulty of selecting an appropriate distribution because calculations do not require an explicit distribution. The only requirement is a random sample of species sensitivities from the universe of possible species sensitivities. The approximate number of species needed to minimize variation around the $\mathrm{HCp}$ estimate can also be obtained by utilizing bootstrap methods.

In the present study, we examined 30 diverse data sets to test the appropriateness of the now prevailing preference for a lognormal distribution for fitting species-sensitivity data; to illustrate an alternate, bootstrap method that imposes no distributional requirements; and to estimate the approximate number of species-sensitivity values required to precisely estimate HC5, HCC10, and HC20 values.

\section{MATERIALS AND METHODS}

\section{Data sources}

No-observed-effect-concentration and EC50-LC50 data were assessed because both are analyzed with species-sensitivity distribution methods. The NOEC data (potassium bichromate; sodium bromide; tetrapropylene benzene sulfonate; 2,4-dichloroaniline; $p$-nitrotoluene; 2,4-dinitro-o-cresol; dimethoate; and pentachlorophenol) were those tabulated in Slooff and Canton [18] with supplemental values from the AQUIRE database [19]. Atrazine EC50-LC50 data were provided by K. Solomon and applied after culling unspecified species from the data set. Diazinon data from Giddings et al. [20] were compiled similarly. Aldrin, copper, chlordane, chromium, dieldrin, endosulfan, and heptachlor data were taken from water-quality criteria documents [21-26] and augmented with the AQUIRE database [19]. Also, data entries for unspecified species were removed from chromium and copper data. Mercury data came from the AQUIRE database except one datum from Khangarot and Ray [27]. Chlorpyrifos data were derived from the AQUIRE database. Methyl parathion, guthion, and malathion data came from the AQUIRE database with expansion from Brandt et al. [28], Cripe [29], Cripe et al. [30], Key et al. [31], Morton et al. [32], and Verschueren [33]. Geometric means were used in analyses if multiple entries for species were found within a data set.

\section{Parametric statistics}

We determined if the lognormal model was generally applicable for fitting species-sensitivity information for each of the 30 data sets (Table 1). After log transformation of effect concentrations, a Shapiro-Wilk's test was performed to ascertain how often the null hypothesis of a (log) normal distribution was rejected $(\alpha=0.05)$ (SAS ${ }^{\circledR}$ Version 6.12 , Proc Univariate [34]). Pearson $\chi^{2}$ statistics were calculated after the 30 data sets were fitted by maximum likelihood methods to lognormal, log-logistic, and Gompertz models (SAS Version 6.12, Proc Probit [34]). Resulting $\chi^{2}$ statistics allowed comparison of data goodness-of-fit for these candidate models: the model with the lowest $\chi^{2}$ statistic was the best. Plots of regression residuals from the candidate models (lognormal, loglogistic, and Gompertz models fit with Proc Probit) were also examined for systematic deviations from the model. Finally, HC5 values and associated 95\% confidence limits were generated with the lognormal model.

\section{Bootstrap statistics}

Alternatives to conventional parametric statistics appeared soon after affordable, powerful computers became widely available [35]. These computationally intensive methods possess several advantages relative to conventional statistics. A relevant advantage in this case is the capacity to generate univariate statistics (i.e., the HC5) and an associated confidence interval without assuming a specific distribution [17]. This method also provides a straightforward way to estimate the number of observations required to minimize the variation about an estimate such as the HC5 $[17,36]$.

Bootstrap estimates of the HC5 and its $95 \%$ confidence limits were produced for the 30 data sets with Resampling Stats Version 4 [37]. For each of the 30 sets, the available data were sampled randomly with replacement to create a resampling data set of 100 observations. These observations were ranked from smallest to largest and the value ranked at the fifth percentile was selected as the HC5. Here and elsewhere, linear interpolation between ranked values was used as required in simulations resampling low numbers of observations. The resampling was repeated to produce 10,000 estimates of HC5. These 10,000 estimates were ranked, and the value corresponding to $50 \%$ was taken as the best estimate of the HC5. The estimates corresponding to 2.5 and $97.5 \%$ were used as the $95 \%$ bootstrap confidence limits. The value ranked at $5 \%$ estimated the concentration protecting $95 \%$ of the species with $95 \%$ certainty, that is, only $5 \%$ or fewer species will be affected 19 out of 20 times at this concentration. Notice that none of these procedures required a specific distribution.

The bootstrap procedure was modified to estimate the number of species-sensitivity values needed to approach the point of minimal variation about a HC5 estimate. The above process was repeated with resampling data sets of size 5 to 100 in increments of 5. The resulting 2.5, 50, and $97.5 \%$ values were plotted against sample size. The confidence interval about the HC5 estimate decreased as sample size increased until the first point of minimal improvement was reached. The sample size at that point approximated the number of observations needed 


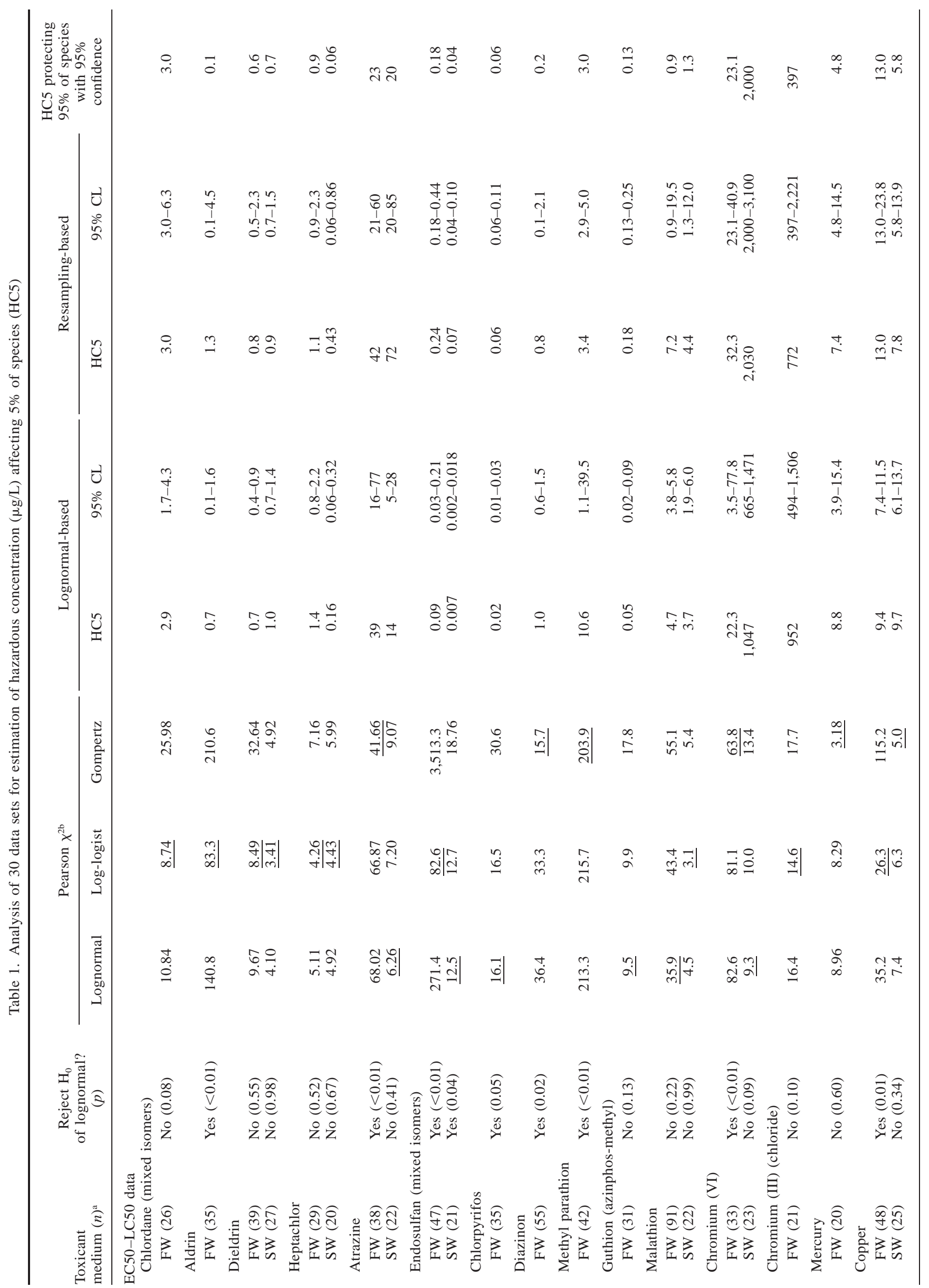




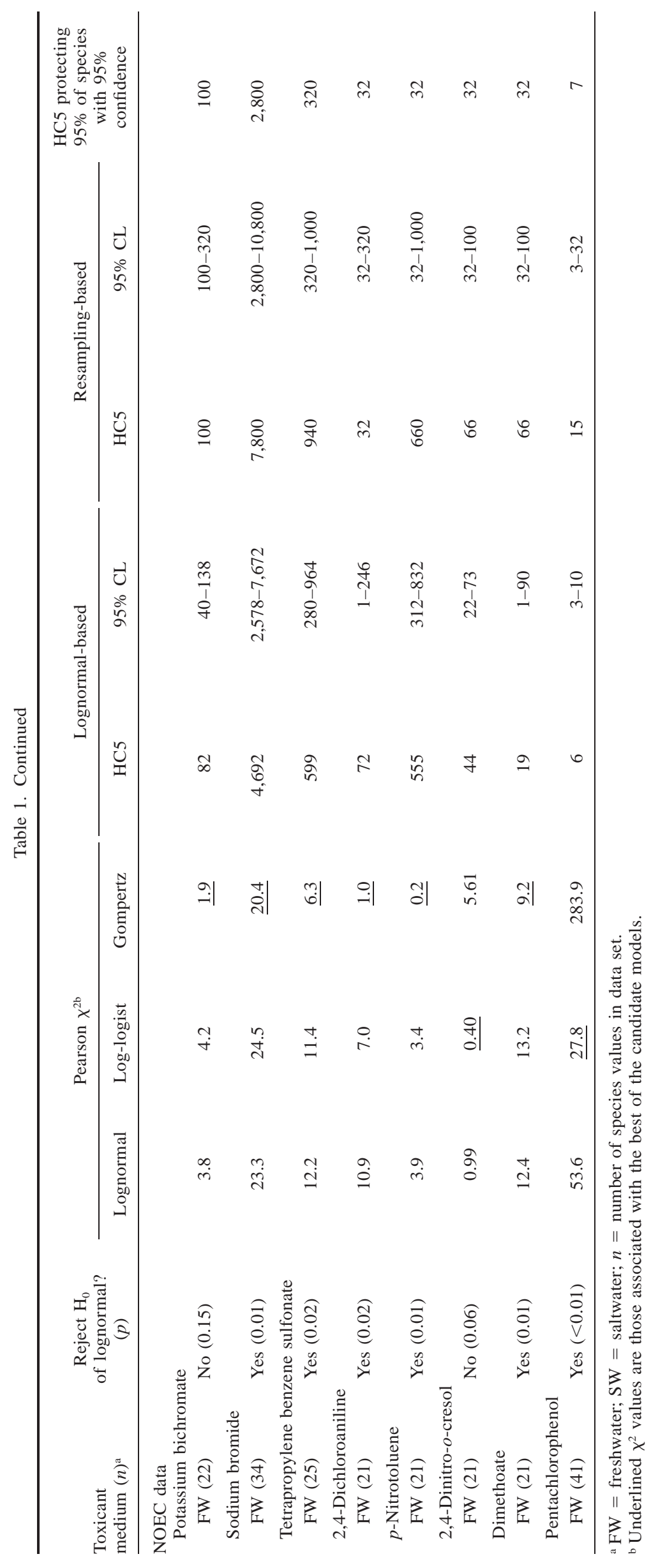




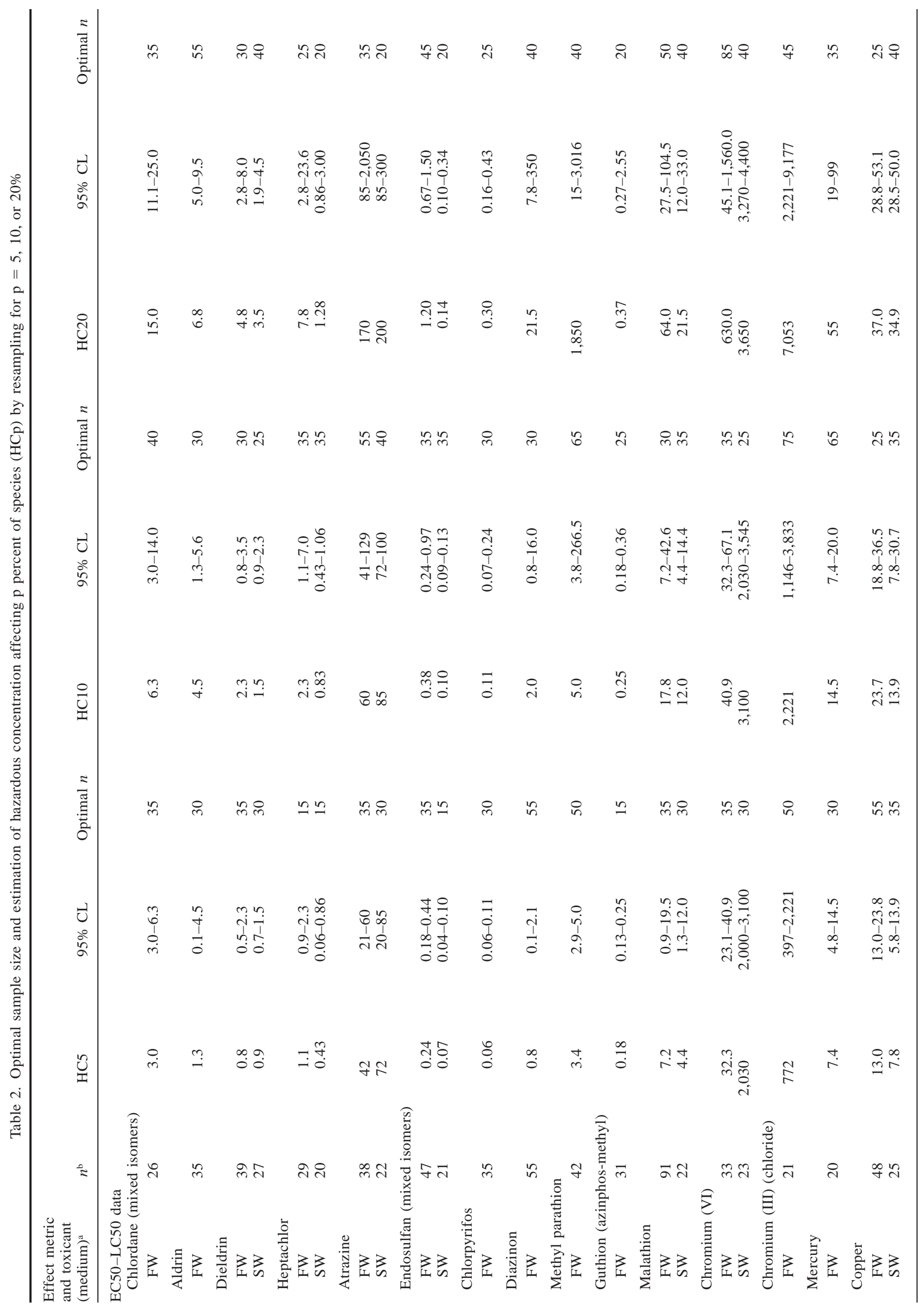




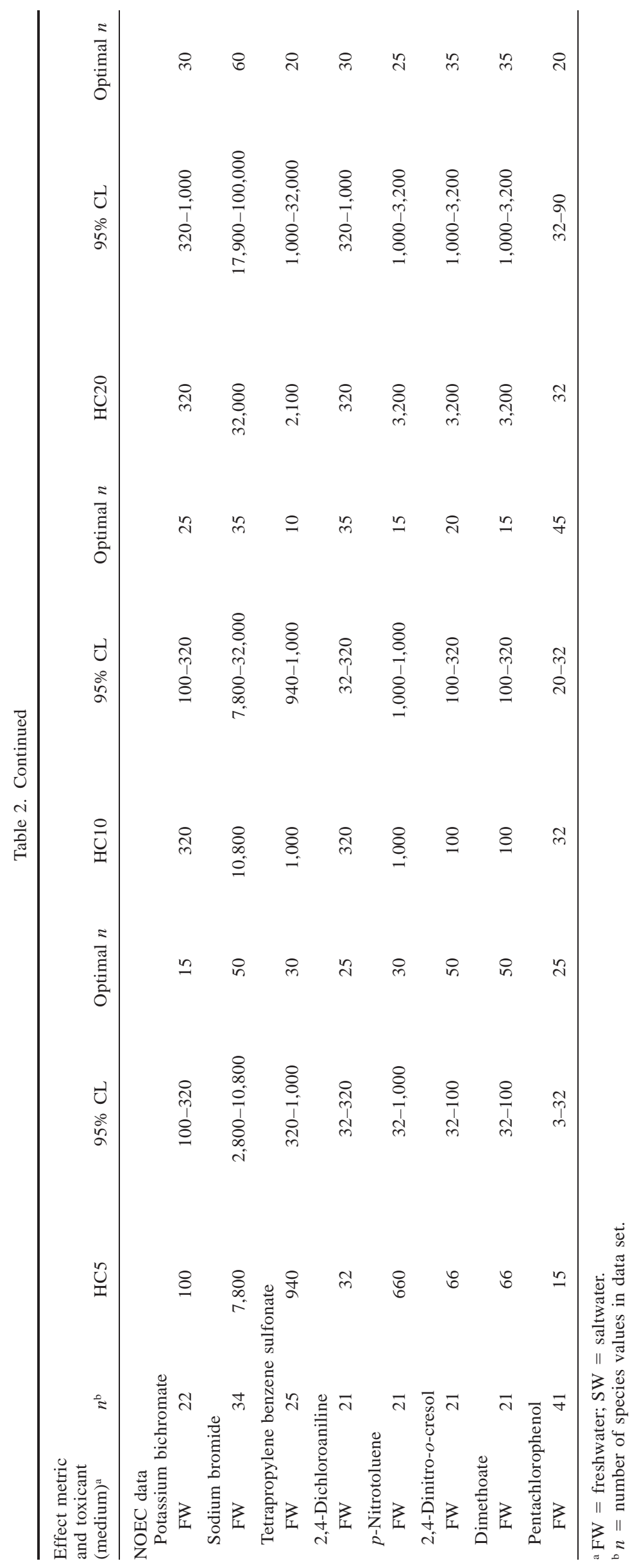




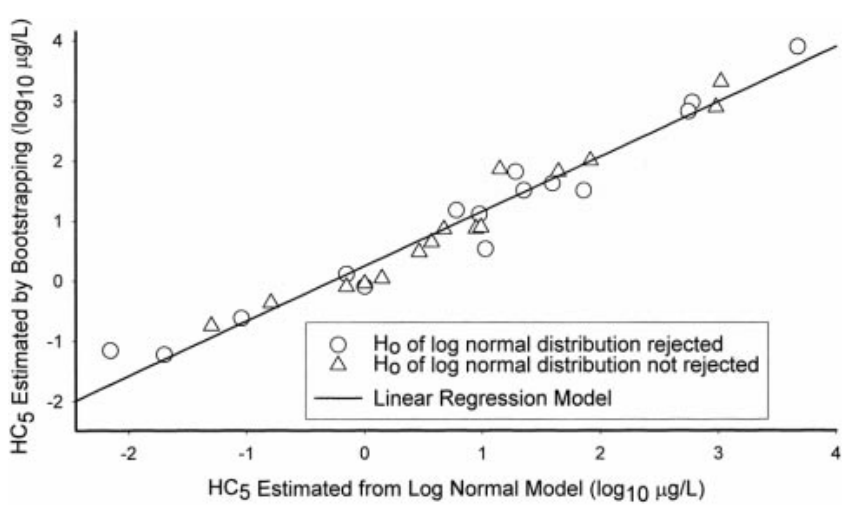

Fig. 1. Predicted hazardous concentration (to $5 \%$ of the species) (HC5) values from the lognormal distribution and bootstrap methods. Results from all 30 data sets are plotted regardless of whether the data failed $(\bigcirc)$ or passed $(\triangle)$ the test for (log) normality.

to obtain minimum variation about HC5. This procedure was applied to the 30 data sets to estimate adequate sample sizes for HC5, HC10, and HC20.

\section{RESULTS}

\section{Adequacy of lognormal model}

The null hypothesis that species-sensitivity data fit a lognormal distribution was rejected for one half of the 30 data sets (Table $1, \alpha=0.05$ ). The $\chi^{2}$ statistics for the EC50 and LC50 data sets indicated that the log-logistic model was the best fit for the data twice as often as the lognormal model. For most of the NOEC data, the Gompertz model fit better than either the lognormal or log-logistic model. Residual plots for models showed distinct patterns for most of the data sets, suggesting systematic variability unaccounted for by the three models. $\mathrm{Cu}$ mulative frequency plots had distinct shifts in slopes (i.e., a multimodal distribution) generally corresponding with transitions among the various taxonomic classes ranked in the data set. The HC5 estimates and associated 95\% confidence limits are provided in Table 1 . Also, a resampling estimate of the conservative $\mathrm{HC} 5$, that concentration protecting $95 \%$ of species with $95 \%$ confidence, is listed in the last column of Table 1.

\section{Comparison of the lognormal and bootstrap methods}

A strong correlation existed between HC5 values predicted with the bootstrap and lognormal model methods (Fig. 1). Least-squares regression (SAS Version 6.12, Proc GLM [34]) produced the model, $\log _{10}$ HC5 from bootstrapping = $0.908\left(\log _{10}\right.$ HC5 from lognormal model $)+0.252$. The standard errors for the estimated slope and intercept were 0.037 and 0.061 , respectively. With perfect correspondence, a slope of one would be expected; however, the slope was significantly lower than one. The model $r^{2}$ of 0.954 indicated a strong relationship between estimates from these two methods. Plots of regression residuals versus log $\mathrm{HC} 5$ based on the lognormal model showed a random pattern, suggesting adequacy of the linear model. No difference was apparent between fit of data sets failing tests of (log) normality (open circles in Fig. 1) and those passing this test (open triangles in Fig. 1). Further, no clear upward or downward bias was found in HCp estimates derived inappropriately from data sets with significant deviation from the assumption of a lognormal distribution.
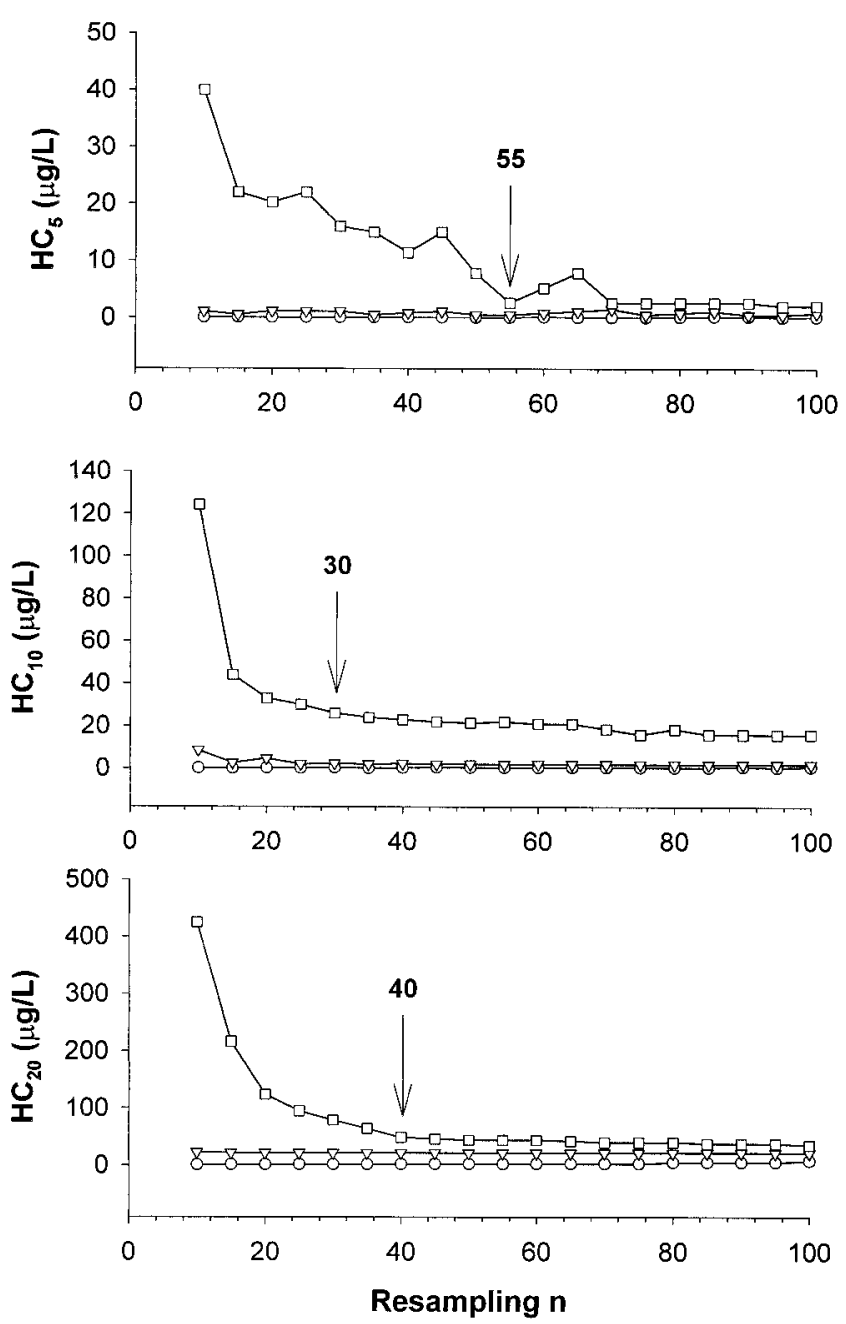

Fig. 2. Curves for estimating sample size for hazardous concentrations to $5 \%$ (HC5), to $10 \%$ ( $\mathrm{HC} 10$ ), and to $20 \%$ (HC20) of the species as illustrated with the diazinon (freshwater) data. The symbols indicate the HCp values ranked at the $50 \%(\nabla), 2.5 \%(\bigcirc)$, and $97.5 \%(\square)$ of the 10,000 values generated by bootstrapping. An arrow indicates the point at which sufficient sample size was reached, with that sample size being indicated above the arrow.

\section{Minimal sample size estimates}

Approximate optimal sample sizes for HC5 estimation ranged from 15 to 55 with a median of 30 species-sensitivity values (Table 2; see also Fig. 2 as an example). Similar sample sizes were needed for $\mathrm{HC} 10$ and $\mathrm{HC} 20$ estimation: estimates ranged from 10 to 75 . No difference was apparent in ranges for EC50-LC50 or NOEC data. These sample sizes are much higher than those recommended as acceptable for regulatory purposes (e.g, four to eight species as detailed in Baker et al. [6]).

\section{DISCUSSION}

Analysis of 30 diverse data sets did not support the general use of a lognormal model to fit species-sensitivity information. One half of the data sets failed the null hypothesis that they came from a lognormal distribution, and plots suggested strong and often multimodal patterns to regression residuals. This was not unexpected because data from distinct taxonomic and ecological groupings are combined in this approach.

The argument could be made that estimates from the lognormal model were sufficient and tend to be slightly more conservative than those from bootstrapping. Also, as suggested 
by results from the small number of data sets explored here, the approach may be relatively robust to violations of normality. However, the defensibility of the species-sensitivity approach would be compromised by the knowledge that a fundamental assumption was frequently violated. It would be difficult to know when errors might emerge because of this violation and how large the error might be. Also, any judgement of robustness would be imprudent based on so few data sets. The resampling method alleviates these ambiguities without sacrificing any ease in computation. A bootstrap approach to generate a concentration at which $95 \%$ of species are protected with $95 \%$ certainty (rightmost column in Table 1) would be as conservative as the lognormal-model-based method without violating any fundamental assumptions.

The species-sensitivity distribution method, despite its potential shortcomings, does provide a pragmatic way of moving ecological risk assessments beyond the hazard quotient. Also, despite its limitations [1], it is a direct means of comparing cumulative exposure concentration distributions to cumulative species-sensitivity distributions. One associated inaccuracy can be excluded by replacing present methods with the simple bootstrap method described herein. The single shortcoming of the bootstrap method-its unfamiliarity to many environmental scientists-is offset by its simplicity. Bootstrapping can be applied with many commercial software packages. Jagoe and Newman [17] list program code to calculate the conservative HC5 method described above.

Approximate numbers of species-sensitivity observations needed to estimate an HCp were much higher than those currently recommended or proposed for regulatory purposes (see Baker et al. [6] as an example). Sufficient information is available for only a few chemicals, for example, atrazine [4]. Regardless, the current inability to meet minimal sample size requirements does not indicate that these pragmatic procedures are invalid. The approximate sample size results derived here are valuable in that they suggest considerable temperance should be exercised during interpretation of results from current procedures. The implied wide variation associated with current sample sizes suggests that the conservative approach should be taken, that is, the concentration protecting $95 \%$ of species with $95 \%$ certainty. Perhaps, required sample sizes would not be as large if estimates were made for specific, sensitive taxonomic groups. Finally, the results provide a target data quality criterion for the future.

Acknowledgement-The comments of M. Mulvey, L. Hall, J. Giddings, J. Meyer, and M. Roberts improved this manuscript significantly and are appreciated. K. Solomon kindly provided the atrazine data.

\section{REFERENCES}

1. Kooijman SALM. 1987. A safety factor for LC50 values allowing for differences in sensitivity among species. Water Res 21:269276.

2. Wagner C, Løkke H. 1991. Estimation of ecotoxicological protection levels from NOEC toxicity data. Water Res 25:1237-1242.

3. Aldenberg T, Slob W. 1993. Confidence limits for hazardous concentrations based on logistically distributed NOEC toxicity data. Ecotoxicol Environ Saf 25:48-63.

4. Solomon KR, et al. 1996. Ecological risk assessment of atrazine in North American surface waters. Environ Toxicol Chem 15:3176.

5. Hall LW Jr, Scott MC, Killen WD. 1998. Ecological risk assessment of copper and cadmium in surface waters of Chesapeake Bay watershed. Environ Toxicol Chem 17:1172-1189.

6. Baker JL, et al. 1994. Aquatic Dialogue Group: Pesticide Risk Assessment and Mitigation. Society of Environmental Toxicology and Chemistry, Pensacola, FL, USA.
7. U.S. Environmental Protection Agency. 1998. Guidelines for ecological risk assessment; notice. Fed Reg 63:26846-26924.

8. Newman MC. 1995. Quantitative Methods in Aquatic Ecotoxicology. CRC, Chelsea, MI, USA.

9. Van Straalen NM, Denneman CAJ. 1989. Ecotoxicological evaluation of soil quality criteria. Ecotoxicol Environ Saf 18:241-251.

10. Pratt JR, Cairns J Jr. 1996. Ecotoxicology and the redundancy problem: Understanding effects on community structure and function. In Newman MC, Jagoe CH, eds, Ecotoxicology. A Hierarchical Treatment. CRC, Boca Raton, FL, USA, pp 347-370.

11. Naeem S, Thompson LJ, Lawler SP, Lawton JH, Woodfin RM. 1994. Declining biodiversity can alter the performance of ecosystems. Nature 368:734-737.

12. Tilman D, Downing JA. 1994. Biodiversity and stability on grasslands. Nature 367:363-365.

13. Tilman D. 1996. Biodiversity: Population versus ecosystem stability. Ecology 77:350-363.

14. Tilman D, Wedlin D, Knops J. 1996. Productivity and sustainability influenced by biodiversity in grassland ecosystems. Nature 379:718-720.

15. Hopkin SP. 1993. Ecological implications of "95\% protection levels" for metals in soil. Oikos 66:137-141.

16. Okkerman PC, Plassche EJVD, Slooff W, Van Leeuwen CJ, Canton JH. 1991. Ecotoxicological effects assessment: A comparison of several extrapolation procedures. Ecotoxicol Environ Saf 21:182-193.

17. Jagoe RH, Newman MC. 1997. Bootstrap estimation of community NOEC values. Ecotoxicology 6:293-306.

18. Slooff W, Canton JH. 1983. Comparison of the susceptibility of 11 freshwater species to 8 chemical compounds. II. (Semi)chronic toxicity tests. Aquat Toxicol 4:271-282.

19. U.S. Environmental Protection Agency. 1997. AQUIRE. AQUatic Toxicity Information REtrival Database. Duluth, MN.

20. Giddings JM, Biever RC, Annunziato MF, Hosmer AJ. 1996. Effects of diazinon on large outdoor pond microcosms. Environ Toxicol Chem 15:618-629.

21. U.S. Environmental Protection Agency. 1980. Ambient water quality criteria for aldrin/dieldrin. EPA 440/5-80-019. Washington, DC.

22. U.S. Environmental Protection Agency. 1980. Ambient water quality criteria for chlordane. EPA 440/5-80-027. Washington, DC.

23. U.S. Environmental Protection Agency. 1980. Ambient water quality criteria for endosulfan. EPA 440/5-80-046. Washington, DC.

24. U.S. Environmental Protection Agency. 1980. Ambient water quality criteria for heptachlor. EPA 440/5-80-052. Washington, DC.

25. U.S. Environmental Protection Agency. 1984. Ambient water quality criteria for chromium. EPA 440/5-84-029. Washington, DC.

26. U.S. Environmental Protection Agency. 1985. Ambient water quality criteria for copper. EPA 440/5-84-031. Washington, DC.

27. Khangarot BS, Ray PK. 1988. Sensitivity of freshwater pulmonate snails, Lymnaea luteola L., to heavy metals. Bull Environ Contam Toxicol 41:208-213.

28. Brandt OM, Fugimura RW, Finlayson BJ. 1993. Use of Neomysis mercedis (Crustacea: Mysidacea) for estuarine toxicity tests. Trans Am Fish Soc 122:279-288.

29. Cripe GM. 1994. Comparative acute toxicities of several pesticides and metals to Mysidopsis bahia and postlarval Penaeus duorarum. Environ Toxicol Chem 13:1867-1872.

30. Cripe GM, Ingley-Guezou A, Goodman LR, Forester J. 1989. Effect of food availability on the acute toxicity of four chemicals to Mysidopsis bahia (Mysidacea) in static exposures. Environ Toxicol Chem 8:330-338.

31. Key PB, Fulton MH, Scott GI, Layman SL, Wirth EF. 1998. Lethal and sublethal effects of malathion on three life stages of the grass shrimp, Palaemonetes pugio. Aquat Toxicol 40:311-322.

32. Morton MG, Mayer FL Jr, Dickson KL, Waller WT, Moore JC. 1997. Acute and chronic toxicity of azinphos-methyl to two estuarine species, Mysidopsis bahia and Cyprinodon variegatus. Arch Environ Contam Toxicol 32:436-441.

33. Verschueren K. 1983. Handbook of Environmental Data on Organic Chemicals, 2nd ed. Van Nostrand Reinhold, New York, NY, USA.

34. SAS Institute. 1988. SAS ${ }^{\circledR} / S T A T$ User's Guide, Version 6.03. Cary, NC, USA.

35. Efron B, Tibshirani R. 1991. Statistical data analysis in the computer age. Science 253:390-395.

36. Manly BFJ. 1992. Bootstrapping for determining sample sizes in biological studies. J Exp Mar Biol Ecol 158:189-196.

37. Resampling Stats. 1995. Resampling Stats User's Guide. Arlington, VA, USA. 